This item was submitted to Loughborough's Research Repository by the author.

Items in Figshare are protected by copyright, with all rights reserved, unless otherwise indicated.

\title{
Homogeneous trees of second order Sturm-Liouville equations: a general theory and program
}

PLEASE CITE THE PUBLISHED VERSION

http://dx.doi.org/10.1016/j.compstruc.2012.03.001

\section{PUBLISHER}

(c) Civil-Comp and Elsevier Ltd.

\section{VERSION}

SMUR (Submitted Manuscript Under Review)

\section{PUBLISHER STATEMENT}

This work is made available according to the conditions of the Creative Commons Attribution-NonCommercialNoDerivatives 4.0 International (CC BY-NC-ND 4.0) licence. Full details of this licence are available at: https://creativecommons.org/licenses/by-nc-nd/4.0/

\section{LICENCE}

CC BY-NC-ND 4.0

\section{REPOSITORY RECORD}

Howson, W.P., and Andrew Watson. 2015. "Homogeneous Trees of Second Order Sturm-liouville Equations: A General Theory and Program”. figshare. https://hdl.handle.net/2134/17510. 


\title{
Homogeneous Trees of Second Order Sturm-Liouville
}

\section{Equations: A General Theory and Program}

\author{
W.P. Howson ${ }^{1}$ and A. Watson ${ }^{2}$ \\ ${ }^{1}$ Cardiff School of Engineering, Cardiff University, UK \\ ${ }^{2}$ Department of Aeronautical and Automotive Engineering, Loughborough University, UK
}

\begin{abstract}
Quantum graph problems occur in many disciplines of science and engineering and they can be solved by viewing the problem as a structural engineering one. The SturmLiouville operator acting on a tree is an example of a quantum graph and the structural engineering analogy is the axial vibration of an assembly of bars connected together with a tree topology. Using the Dynamic Stiffness Matrix method the natural frequencies of the system can be determined which are analogous to the eigenvalues of the quantum graph. Theory is presented that yields exact solutions to the SturmLiouville problem on homogeneous trees. This is accompanied by an extremely efficient and compact computer program that implements the theory. An understanding of the former is enhanced by recourse to a structural mechanics analogy, while the latter program is fully annotated and explained for those who might wish to extend its capability. In addition, the use of the program as a 'black box' is fully described and a small parametric study is undertaken to confirm the accuracy of the approach and indicate its range of application including to the computation of negative eigenvalues.
\end{abstract}


Keywords: Quantum Graphs; Sturm-Liouville equation; homogeneous trees; subtreeing; transcendental eigenvalue problem; Dynamic Stiffness Matrix; Negative Eigenvalues; Wittrick-Williams algorithm.

\section{Introduction}

Quantum graphs occur widely in many areas of science and engineering such as mathematics, chemistry, physics, nanotechnology and photonic crystals. The subject is a substantial one and reference [1] is a good introduction to the subject. A quantum graph is defined as a graph with a differential operator acting. A graph is a set of connected edges with each edge having a pair of vertices. The edges have a defined length, $L$, and the graph is then defined to be metric. Quantum graphs are one dimensional structures. Figure 1 shows a non-cyclic graph, namely a tree, and a cyclic graph.

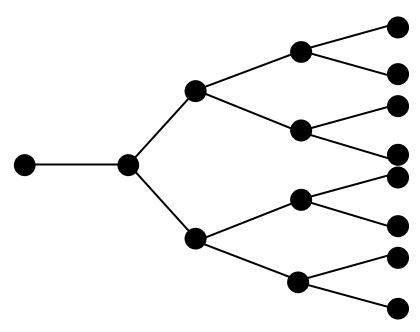

(a)

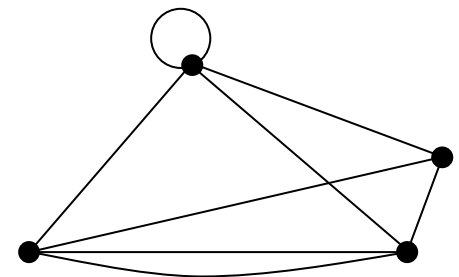

(b)

Figure 1: Graph (a) Non-cyclic graph (tree); (b) Cyclic graph.

Consideration is given to the mathematical problem of calculating the eigenvalues of homogeneous trees, as shown in Figure 1(a), for which there is much current interest [2- 
4]. The tree in question comprises a single trunk that divides into $b$ branches at its tip, with each branch dividing into $b$ sub-branches ad infinitum. Examples of typical trees, showing their level and branching numbers, are given in Figure 2. The second order Sturm-Liouville equation, defined by Equation (1), is then used to describe each branch and these equations define a matrix that can be used to describe the tree.

$$
-\frac{d}{d x}\left(p \frac{d y}{d x}\right)+q y=\lambda w y
$$

where $x \in[a *, b *), a *<b *<\infty$, when $\mathrm{a}^{*}$ is a regular point and $\mathrm{b}^{*}$ is either regular or singular, under separated self adjoint boundary conditions. The parameters $p, q$ and $w$ are all real valued, positive constants. $\lambda$ is the eigenvalue parameter. Assigning the values of $p=w=1$ and $q=0$ reduces the general $2^{\text {nd }}$ order Sturm-Liouville equation to the LaPlace equation. Equation 1 is exactly analogous to the axial vibration equation of a non-uniform bar on a non-uniform elastic foundation as follows

$$
-\frac{d}{d x}\left(E A \frac{d y}{d x}\right)+k y=\omega^{2} m y
$$

where $E A$ is the extensional rigidity of the bar, $k$ is the stiffness per unit length of the elastic foundation and $\mathrm{m}$ is the mass per unit length. $\omega$ is natural frequency parameter.

Initially Equation (1) is developed into an ‘edge’ matrix, which describes each branch of the tree in a way that enables the branches to be linked together at vertices to form the tree. This linking is analogous to the way elements are joined together at nodes to form structures when using the stiffness technique. The structural mechanics analogy is further enhanced by noting the correspondence between the 'edge' matrix and the exact dynamic stiffness matrix of the axially vibrating, uniform bar. The relationship is fully explained later, but means that the eigenvalues of the mathematical 
problem correspond precisely to natural frequencies when the branches are replaced by axially vibrating bars. This gives considerable insight into the problem when associating a physical relevance to the results determined.

An annotated listing of a FORTRAN 77 computer program that implements the theory and builds the required tree structure is presented and its use is fully explained. The exact approach adopted necessitates the solution of a transcendental eigenvalue problem. This is achieved using the Wittrick-Williams algorithm, which guarantees that the required eigenvalues are converged upon to any desired accuracy with the certain knowledge that none have been missed. However, the application of the WittrickWilliams algorithm in this case is somewhat unusual due to the presence of deeply nested sub-structures that are used to describe trees that can easily have in excess of $10^{12}$ branches.

A parametric study is finally undertaken that confirms those results that are available in the literature and extends them by considering the effects of changing the parameters $L, p, q$ and $w$. 


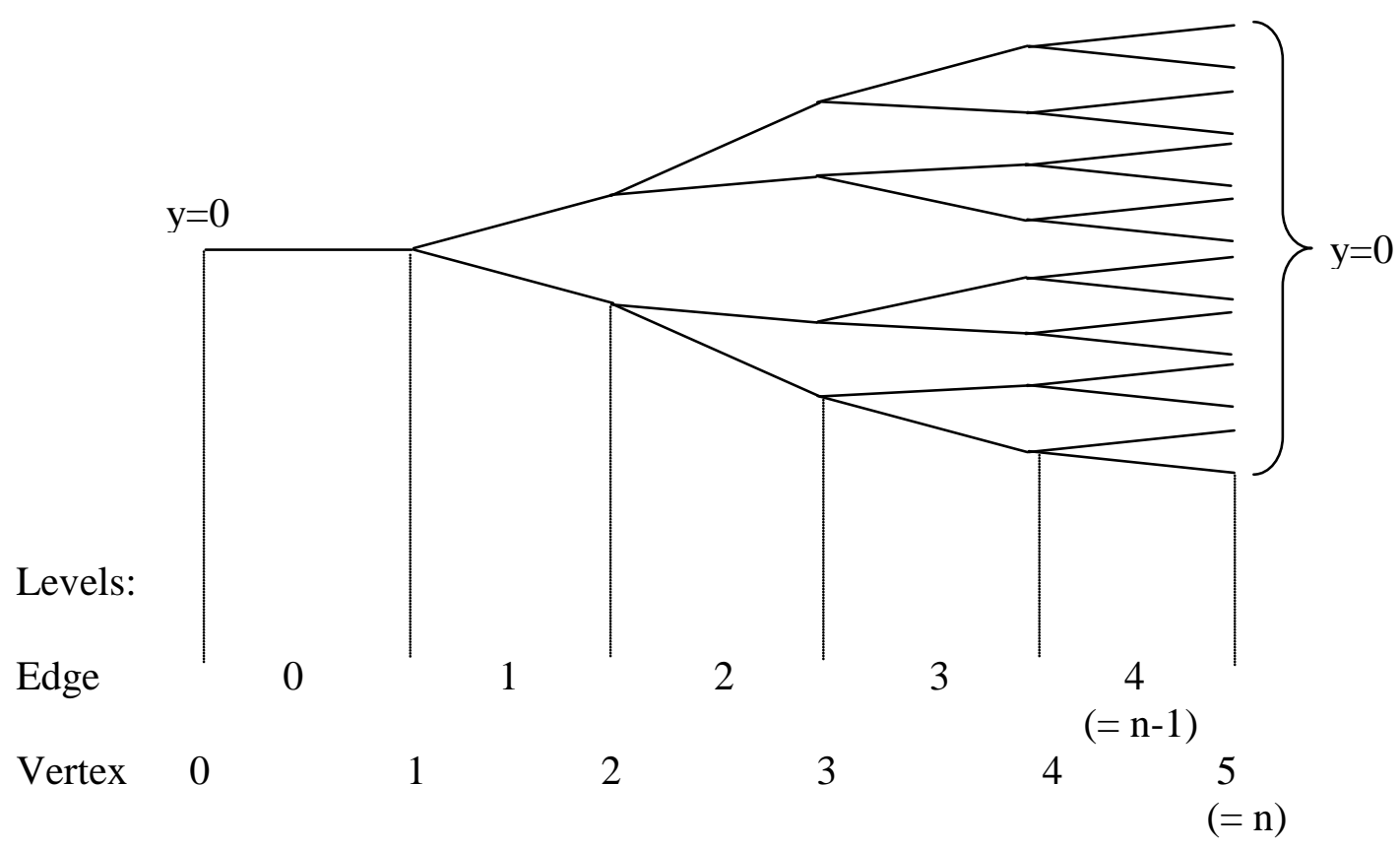

(a) A five level, two branching tree i.e. $n=5, b=2$ with Dirichlet boundary conditions

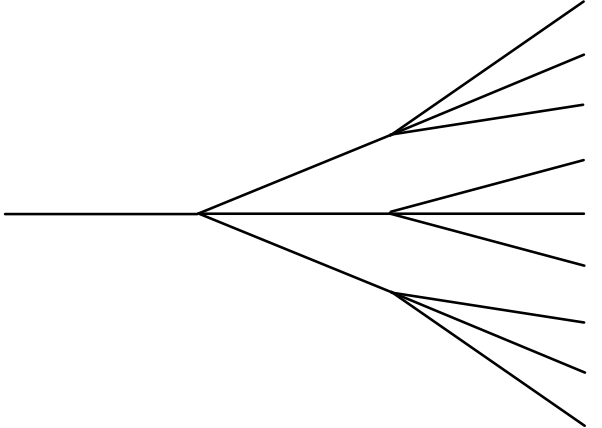

(b) $n=3, b=3$

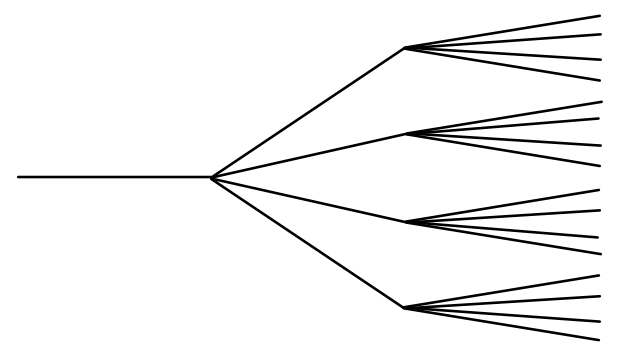

(c) $n=3, b=4$

Figure 2: The topology of typical trees showing edge and vertex levels together with their branching number

\section{Previous Work}

There has been much interest within the mathematical community [2-8] for developing algorithms and numerical software to compute the eigenvalues and eigenvectors of the classical Sturm-Liouville problem. Further algorithms and programs have been developed so that both periodic and linked boundary conditions can be accommodated 
[9]. Furthermore, in both the regular and singular cases, precision can be given to these results by enclosing them in intervals whose bounds can be proven to be correct [10]. There is also much current interest in the problem of homogeneous trees $[2,3]$ with the recent work of Sobolev \& Solomyak [4] being of particular relevance because they show that for an infinite tree, the eigenvalues of the free Laplacian in one dimension form bands of absolutely continuous spectra with eigenvalues of infinite multiplicity in the gaps. For other operators on a homogeneous tree, having similar nature, the bandgap structure of the spectrum was established earlier by Carlson [11] with the Hill operator. Sobolev \& Solomyak [4] also consider the effect on the complete spectrum of introducing a small perturbation in the form of a real-valued potential, $q$. These issues were recently addressed by Williams et. al $[12,13]$ in order to develop exact solutions for the distribution of eigenvalues on homogeneous trees defined by Equation (1). The present paper provides; a non-dimensional formulation of the theory given in [12,13]; a compact computer program to implement it; and a simple way of modifying the eigenvalue to account for variations in $L, p, q$ and $w$ of Equation (1).

\section{Theory}

\subsection{The edge matrix}

The theory presented in this section relates to the general form of the classical secondorder Sturm-Liouville equation shown in equation (1). A more rigorous definition of the problem is given in references [12,13]. Introducing the non-dimensional parameter

$$
\xi=\frac{x}{L}
$$


enables Equation (1) to be written as

$$
-\frac{d^{2} y}{d \xi^{2}}=\alpha^{2} y
$$

where $\alpha^{2}=\frac{(\lambda w-q) L^{2}}{p}$. Equation (4) then is the problem reduced to the one

dimensional LaPlacian operator acting on the tree. The general solution of Equation (4) is well known to be

$$
y=A \cos \alpha \xi+B \sin \alpha \xi
$$

Substituting the boundary conditions

$$
y=y_{1} \quad \text { at } \quad \xi=0 \quad \text { and } \quad y=y_{2} \quad \text { at } \quad \xi=1
$$

gives

$$
A=y_{1} \quad \text { and } \quad B=y_{2} \operatorname{cosec} \alpha-y_{1} \cot \alpha
$$

Substituting Eqs. (7) into Equation (5) gives

$$
y=y_{1} \cos \alpha \xi+\left(y_{2} \operatorname{cosec} \alpha-y_{1} \cot \alpha\right) \cos \alpha \xi
$$

and hence

$$
\frac{d y}{d \xi}=-\alpha\left[y_{1} \sin \alpha \xi+\left(y_{1} \cot \alpha-y_{2} \operatorname{cosec} \alpha\right)\right]
$$

Denoting

$$
\frac{d y}{d \xi}=y_{1}^{\prime} \quad \text { at } \quad \xi=0 \quad \text { and } \quad \frac{d y}{d \xi}=y_{2}^{\prime} \quad \text { at } \quad \xi=1
$$

gives

$$
\left[\begin{array}{c}
-y_{1}^{\prime} \\
y_{2}^{\prime}
\end{array}\right]=\frac{\alpha}{\sin \alpha}\left[\begin{array}{cc}
\cos \alpha & -1 \\
-1 & \cos \alpha
\end{array}\right]\left[\begin{array}{l}
y_{1} \\
y_{2}
\end{array}\right]
$$


Equation (11) is the matrix relationship stemming from Equation (1) that defines each branch of the tree. In the current mathematical context, and to align it with previous work $[12,13]$, it will be referred to as the 'edge' equation that links any two vertices. Vertices at the root of the tree and the tips of the top branches are then subject to any combination of Dirichlet $(y=0)$ or Neumann $(d y / d \xi=0)$ boundary conditions.

Figure 2 typifies the tree topology, shows Dirichlet boundary conditions and defines the edge and vertex levels, together with $n$ and $b$, the number of vertex levels and branching number, respectively. All trees are classified as repetitive or nonrepetitive, depending upon whether or not the edge properties at all levels are identical, and such trees are sub-divided into uniform or non-uniform, depending upon whether or not $L, p, q$ and $w$ are all constant. Hence, a repetitive uniform tree is a homogeneous one, whereas a repetitive non-uniform tree is not. This paper deals only with homogeneous trees.

The remainder of this paper deals with Equation (11) explicitly, although it is extremely helpful to note a structural mechanics analogy that aids interpretation of results and is crucially important when arguing fundamental properties of the system [12,13]. Let Equation (11) be multiplied throughout by $p / L$. It then becomes the equation for axial vibration of a uniform bar if the parameters have the following meanings attributed to them; $\lambda=\omega^{2}, p=E A, q=k$ and $w=m$, where $\omega$ is the angular frequency, $E A$ is the axial rigidity, $k$ is the stiffness/unit length of axial elastic support and $m$ is the mass/unit length. Hence $\alpha^{2}$ takes on the familiar form $\alpha^{2}=\left(m \omega^{2}-k\right) L^{2} / E A$. The terms on the left-hand side of Equation (11) then become - $E A y_{1}^{\prime} / L$ and $E A y_{2}^{\prime} / L$, which are the axial forces acting to the right, at the left and 
right-hand ends of the bar and $y_{1}$ and $y_{2}$ are the corresponding axial displacements. When edges are interpreted as such bars, Figure 2 represents the free axial vibration problem of tree structures so long as it is understood that for this vibration problem Figure 2 is foreshortened vertically, such that all of the bars are horizontal and hence colinear. Such a structure is not a practical one, because it would involve all the bars at any level occupying the same space, but is nevertheless valid for its current use as an analogy. In this analogy the boundary conditions, $y=0$ or $y^{\prime}=0$ at vertices, correspond to clamped and free ends of bars, respectively.

\subsection{The Wittrick-Williams algorithm}

The Wittrick-Williams algorithm for converging on the roots of transcendental eigenvalue problems is fully defined in references [12,13] and can be stated in a form that is appropriate for use with the sub-systems used herein as

$$
J=\sum J_{e}+\sum s\left\{\mathbf{A}_{s}\right\}+s\{\mathbf{A}\}
$$

where $J$ is the number of eigenvalues of the tree exceeded by some trial value of the eigenparameter, $\alpha^{*} ; J_{e}$ is the number of eigenvalues of an edge, with Dirichlet boundary conditions, that would still be exceeded by $\alpha^{*}$, and the summation extends over all edges comprising the tree; $s\left\{\mathbf{A}_{s}\right\}$ is the sign count of a sub-tree matrix, $\mathbf{A}_{s}$, where the summation is taken over all sub-trees; and $s\{\mathbf{A}\}$ is the sign count of the reduced tree matrix, see below. The sign count of a matrix is defined as the number of negative elements on the leading diagonal of the upper triangular form of the matrix when $\alpha=\alpha^{*}$ by the standard form of Gauss elimination without row interchanges. 


\subsection{Application of the Wittrick-Williams algorithm}

Consider the three level, two branching (binary) tree shown in Figure 3. The analysis begins by considering the two most deeply nested sub-trees, which are in edge level $i=$ 2, although only one needs to be analysed because they are identical.

Levels:

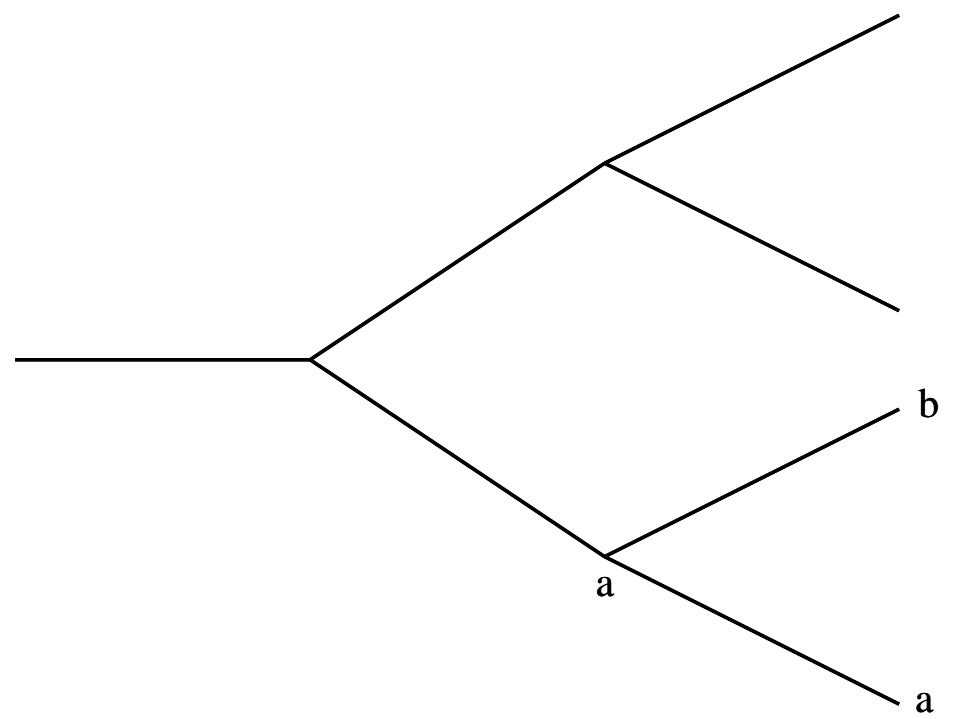

Edge

$\begin{array}{lllll}\text { Vertex } & 0 & 1 & 2 & 3\end{array}$

Figure 3: A three level, binary tree in which one of the two most deeply nested subtrees at edge level $i=2$ is annotated and represents the start point for analysis.

The necessary eigenvalue relationship for a single edge, evaluated at a trial eigenparameter, $\alpha^{*}$, is given by Equation (11) as

$$
\left[\begin{array}{l}
0 \\
0
\end{array}\right]=\left[\begin{array}{ll}
A_{1} & A_{2} \\
A_{2} & A_{1}
\end{array}\right]\left[\begin{array}{l}
y_{1} \\
y_{2}
\end{array}\right]
$$


where $A_{1}=\cot \alpha^{*}$ and $A_{2}=-\operatorname{cosec} \alpha^{*}$. When Dirichlet boundary conditions are imposed, $y_{1}=y_{2}=0$, and the required eigenvalues correspond to those values of $\alpha^{*}$ for which $A_{1}=A_{2}=\infty$. i.e. when $\sin \alpha^{*}=0$. Hence, the number of eigenvalues passed is given by

$$
\operatorname{int}\left(\alpha^{*} / \pi\right)
$$

where int $\left(\alpha^{*} / \pi\right)$ is the highest integer $<\alpha^{*} / \pi$. Since there are $b^{i}$ identical edges at edge level $i$ in the tree, the contribution to $J_{e}$ is given by

$$
J_{e i}=b^{i} \times \operatorname{int}\left(\alpha^{*} / \pi\right)
$$

In similar fashion, a typical sub-tree matrix can be developed from Equation (13) and using the notation of Figure 3 as

$$
\left[\begin{array}{l}
0 \\
0 \\
0
\end{array}\right]=\left[\begin{array}{ccc}
A_{1} & 0 & A_{2} \\
0 & A_{1} & A_{2} \\
A_{2} & A_{2} & b A_{1}
\end{array}\right]\left[\begin{array}{l}
y_{3 a} \\
y_{3 b} \\
y_{2 a}
\end{array}\right]
$$

The analysis then proceeds to eliminate the vertices at vertex level three by performing Gauss elimination, but arresting the process after the first two rows have been pivotal. This leaves the reduced sub-tree parameter $A_{s}$, which corresponds to the displacement, $y_{2 a}$, at the next vertex level down. i.e.

$$
\left[\begin{array}{l}
0 \\
0 \\
0
\end{array}\right]=\left[\begin{array}{ccc}
A_{1} & 0 & A_{2} \\
0 & A_{1} & A_{2} \\
0 & 0 & A_{s}
\end{array}\right]\left[\begin{array}{l}
y_{3 a} \\
y_{3 b} \\
y_{2 a}
\end{array}\right]
$$

where

$$
A_{s}=b\left(A_{1}^{2}-A_{2}^{2}\right) / A_{1}
$$


The sign count $s\left\{\mathbf{A}_{s}\right\}$ for the current sub-tree is either zero if $A_{1}>0$, or $b$ if $A_{1} \leq 0$.

The contribution from all such sub-trees at current edge level $i$ is therefore either zero or $b^{i}$.

The form of the matrix relationship corresponding to Equation (17) for level $i-1$, and all subsequent sub-tree levels for $n>3$, is

$$
\left[\begin{array}{l}
0 \\
0 \\
0
\end{array}\right]=\left[\begin{array}{ccc}
A_{1}^{*} & 0 & A_{2} \\
0 & A_{1}^{*} & A_{2} \\
0 & 0 & A_{s}^{*}
\end{array}\right]\left[\begin{array}{l}
y_{3 a} \\
y_{3 b} \\
y_{2 a}
\end{array}\right]
$$

where $A_{1}^{*}=A_{1}+A_{s}, A_{s}$ is the sub-tree parameter from the previous nesting level and

$$
A_{s}^{*}=b\left(A_{1} A_{1}^{*}-A_{2}^{2}\right) / A_{1}^{*}
$$

The tree is progressively reduced by the process described above until only the trunk is left. The trunk matrix, or reduced tree matrix, can then be deduced as

$$
\left[\begin{array}{l}
0 \\
0
\end{array}\right]=\left[\begin{array}{cc}
A_{1}^{*} & A_{2} \\
A_{2} & A_{1}
\end{array}\right]\left[\begin{array}{l}
y_{1} \\
y_{0}
\end{array}\right]
$$

The process is terminated by imposing the boundary condition at the root, performing Gauss elimination and establishing the sign count, which in this case is equal to $s\{\mathbf{A}\}$.

An annotated listing of a FORTRAN 77 computer program to implement the above theory is given in Appendix A. The program uses $\alpha$ as the eigenparameter, although it could equally well have used $\lambda$, since they are related via

$$
\lambda=\frac{p}{w L^{2}} \alpha^{2}+\frac{q}{w}
$$




\section{Examples}

A general overview of the band-gap spectrum of eigenvalues on homogeneous trees has been given by Williams et al. [12]. Such results are typified by Figure 4, which shows the first five repeating portions of the infinite band-gap spectra for the family of trees shown in Figure 2. All possible eigenvalues are contained within the bands (the bullet shaped envelopes) except for a high multiplicity eigenvalue that occurs at the midpoint of each gap. As $n \rightarrow \infty$, the bands become fully populated and their widths, established theoretically by Sobolev and Solomyak [4], approach the limits indicated by the dashed lines.

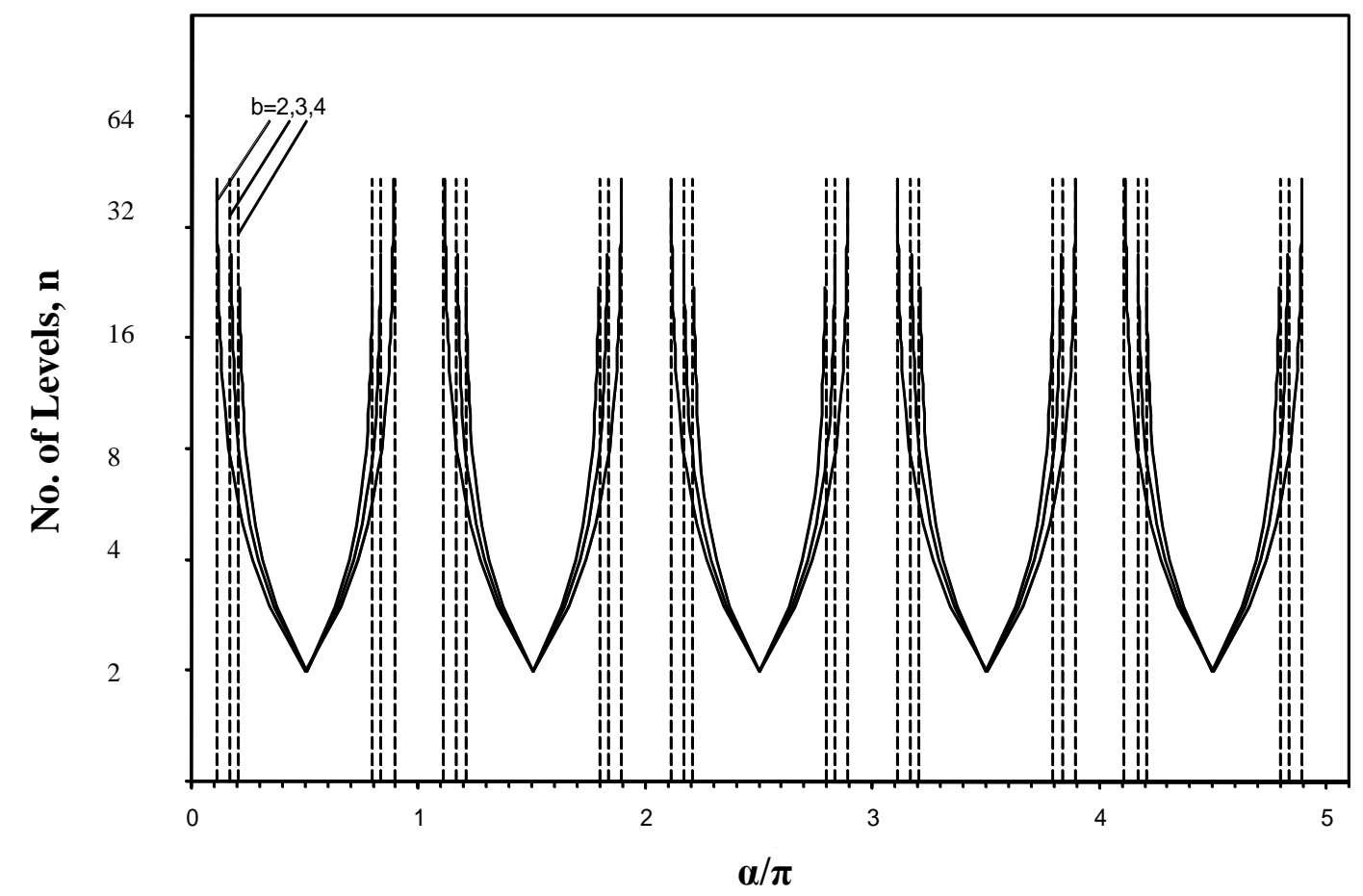

Figure 4: The first five repeating portions of the infinite band-gap spectra for the family of trees shown in Figure 2 when all edges are identical. The Sobolev and Solomyak bounds are shown dashed [4]. 


\subsection{Trees with $L=p=w=1, q=0$ and Dirichlet boundary conditions}

The spectrum of eigenvalues for a homogeneous tree comprising a finite number of levels is made up of discrete values i.e. the band is not fully populated. Figure 5 shows the set of eigenvalues that populate the first band of the spectrum of Figure 4 for trees with $b=3$ and $n=1$ to 8 . Table 1 then gives their values ( $n=2$ to 8 ), together with their multiplicities. It can be seen from either source, that as the value of $n$ increases, new eigenvalues appear and these new values continue to be present for higher values of n. Sobolev and Solomyak [3] proved that the spectrum is fully populated, within the bounds shown, for a tree with an infinite number of levels. For the $b=3$ tree, the lower bound has a value of 0.166667 and the upper bound has a value of 0.833333 . The eigenvalues in the band corresponding to the $n=8$ tree comprise the first twenty one eigenvalues, while the twenty second lies at the midpoint of the gap between bands one and two. Both Figure 5 and Table 1 show that those trees with $n<8$ have fewer eigenvalues in the first band. e.g. the first eigenvalue for the $n=4$ tree is the fifth eigenvalue of the $n=8$ tree.

The multiplicities can also be seen to grow very quickly. Equations have been derived that describe the growth of the multiplicities with increase in $n$ [12]. The eigenvalue that exists in the middle of the gap grows especially fast and its multiplicity is given by $\mathrm{M}_{1}$, where

$$
M_{1}=b^{n-1}
$$

When $n=8$ the multiplicity for this eigenvalue has grown to 2187. Although not shown, the growth of multiplicities is identical for eigenvalues in the higher bands. The 
structure of each band is also seen to be symmetric about its centre, which has a normalised abscissa value of 0.5 for the first band.

The number of eigenvalues in the band is given by $N_{B}$, where

$$
N_{B}=\frac{b^{n-1}-1}{b-1}
$$

For the $n=8$ tree, the number of eigenvalues in the band and the gap is 1093 and 2187, respectively, which together gives a total of 3280 eigenvalues.

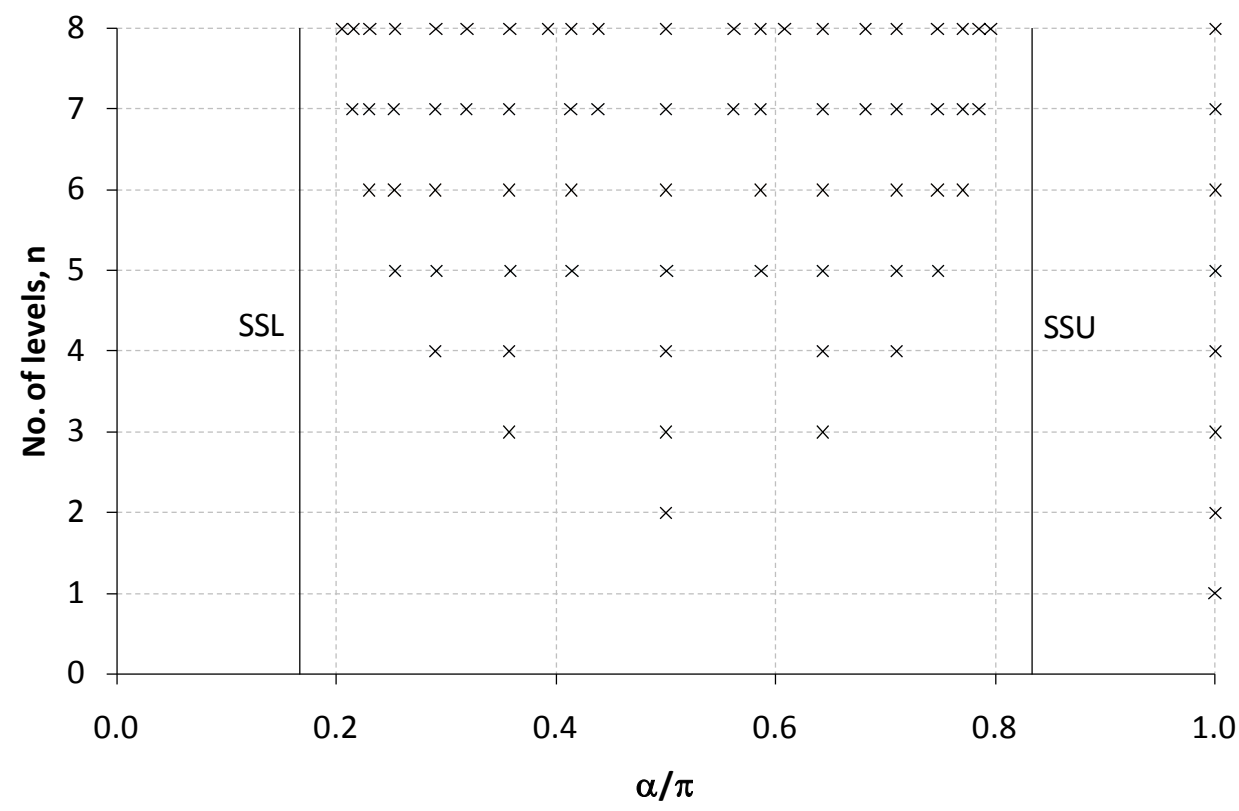

Figure 5: Discrete eigenvalues for a tree with $b=3 ; L=p=w=1 ; q=0$ and Dirichlet $(y=0)$ boundary conditions at each end of the tree. The two solid lines SSL and SSU are theoretical lower/upper bounds, respectively, obtained by Sobolev and Solomyak [4]

\subsection{Variation of $L, p, w$ and $q$}

A parametric study has been conducted to investigate the effect of varying each parameter individually from their default values of $L=p=w=1$ and $q=0$. Dirichlet $(y$ $=0$ ) boundary conditions are imposed at each end of the tree throughout. 
Table 1: Numerical values of the normalised eigenvalues described in Figure 5, together with their multiplicities

\begin{tabular}{|c|c|c|c|c|c|c|c|c|c|}
\hline \multicolumn{10}{|c|}{$n$} \\
\hline $\begin{array}{l}\text { Eig. } \\
\text { No. }\end{array}$ & $\alpha / \pi$ & 8 & 7 & 6 & 5 & 4 & 3 & 2 & 1 \\
\hline 1 & 0.204778 & 1 & & & & & & & \\
\hline 2 & 0.215086 & 2 & 1 & & & & & & \\
\hline 3 & 0.230053 & 6 & 2 & 1 & & & & & \\
\hline 4 & 0.252903 & 18 & 6 & 2 & 1 & & & & \\
\hline 5 & 0.290215 & 55 & 18 & 6 & 2 & 1 & & & \\
\hline 6 & 0.318440 & 2 & 1 & & & & & & \\
\hline 7 & 0.357451 & 168 & 56 & 19 & 6 & 2 & 1 & & \\
\hline 8 & 0.392474 & 1 & & & & & & & \\
\hline 9 & 0.413764 & 18 & 6 & 2 & 1 & & & & \\
\hline 10 & 0.438273 & 2 & 1 & & & & & & \\
\hline 11 & 0.500000 & 547 & 182 & 61 & 20 & 7 & 2 & 1 & \\
\hline 12 & 0.561727 & 2 & 1 & & & & & & \\
\hline 13 & 0.586236 & 18 & 6 & 2 & 1 & & & & \\
\hline 14 & 0.607526 & 1 & & & & & & & \\
\hline 15 & 0.642550 & 168 & 56 & 19 & 6 & 2 & 1 & & \\
\hline 16 & 0.681560 & 2 & 1 & & & & & & \\
\hline 17 & 0.709784 & 55 & 18 & 6 & 2 & 1 & & & \\
\hline 18 & 0.747097 & 18 & 6 & 2 & 1 & & & & \\
\hline 19 & 0.769947 & 6 & 2 & 1 & & & & & \\
\hline 20 & 0.784914 & 2 & 1 & & & & & & \\
\hline 21 & 0.795221 & 1 & & & & & & & \\
\hline 22 & 1.000000 & 2187 & 729 & 243 & 81 & 27 & 9 & 3 & 1 \\
\hline
\end{tabular}

\subsubsection{Variation of $q$}

The effect of introducing $q$ causes a shift in the spectrum and this can be seen in Figure

6. It will be recalled from the end of Section 3.1, that according to the structural mechanics analogy, $q$ is equal to the stiffness, $k$, of the elastic medium constraining the motion of an equivalent bar. Hence an increase in $k$ would cause the natural frequencies to increase. Furthermore the relationship $\alpha^{2}=\frac{(\lambda w-q) L^{2}}{p}$ shows that the lower eigenvalues will be shifted further than the higher ones. This can be seen in Figure 6, where the first eigenvalue for $q=0$ has a normalised value of approximately 0.2 , while 
the highest has a value of approximately 0.8. Compare this with the $q=8$ equivalents of approximately 0.9 and 1.2 .

In structural mechanics natural frequencies are typically positive values. However in quantum graph theory negative eigenvalues do occur [14-16] and their computation is not restricted by theory presented. Watson and Howson [17] show that the parameter $q$ of Equation (1) is not restricted to being negative and computation of negative eigenvalues is then possible as seen in Figure 6. By using a negative value of $q$ results in a shift in the spectrum to the left. The structural mechanics analogy is that an elastic medium with a negative stiffness will cause the natural frequencies to shift to the left and become negative. Note that the abscissa values of Figure 6 to the left of the origin are imaginary numbers and hence need to be multiplied by $-\sqrt{ }(-1)$. The squaring of these values then leads to negative numbers.

\subsubsection{Variation of $L, p$ and $w$}

The relationship $\alpha^{2}=\frac{(\lambda w-q) L^{2}}{p}$ reduces to the three following variants when the parameters are varied independently

$$
\alpha^{2}=\lambda L^{2}, \quad \alpha^{2}=\lambda / p \quad \text { and } \quad \alpha^{2}=\lambda w \quad \text { (25a,b,c) }
$$

From which it follows that the variations in $L, p$, and $w$ are linked as follows

$$
\delta p=1 / \delta w=1 / \delta\left(L^{2}\right)
$$

leading to the variations shown in Figure 7. By analogy $p=E A$ and $w=m$, hence increasing $p$ leads to an increase in the eigenvalue, while increasing $L$ and $m$ leads to a decrease. 


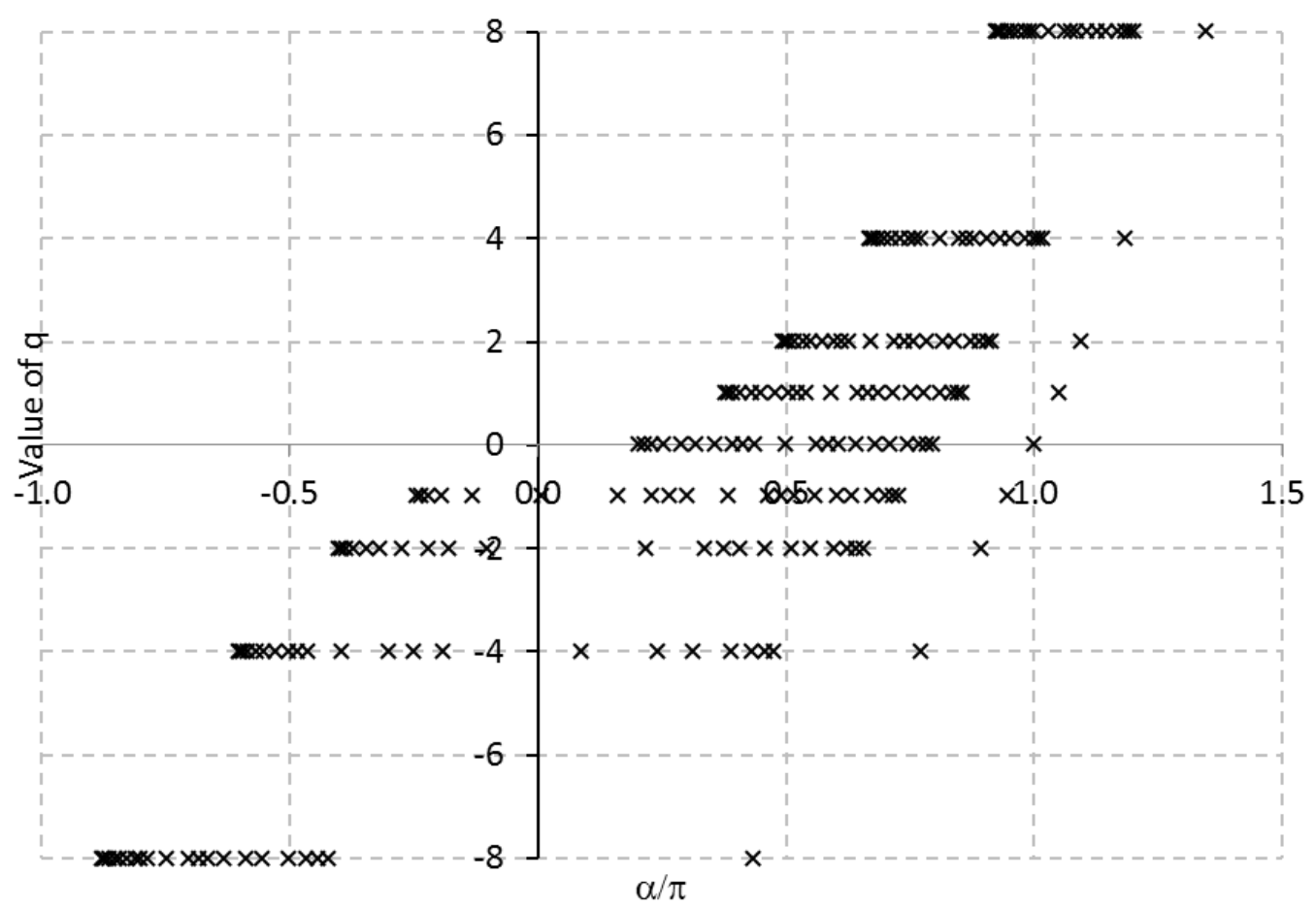

Figure 6: Effect of varying $q$ on the eigenvalues of a tree with $b=3$ with $L=p=w=1$ and Dirichlet / Dirichlet boundary conditions [17]. The abscissa values to the left of the origin are imaginary numbers and hence need to be multiplied by $-\sqrt{ }(-1)$.

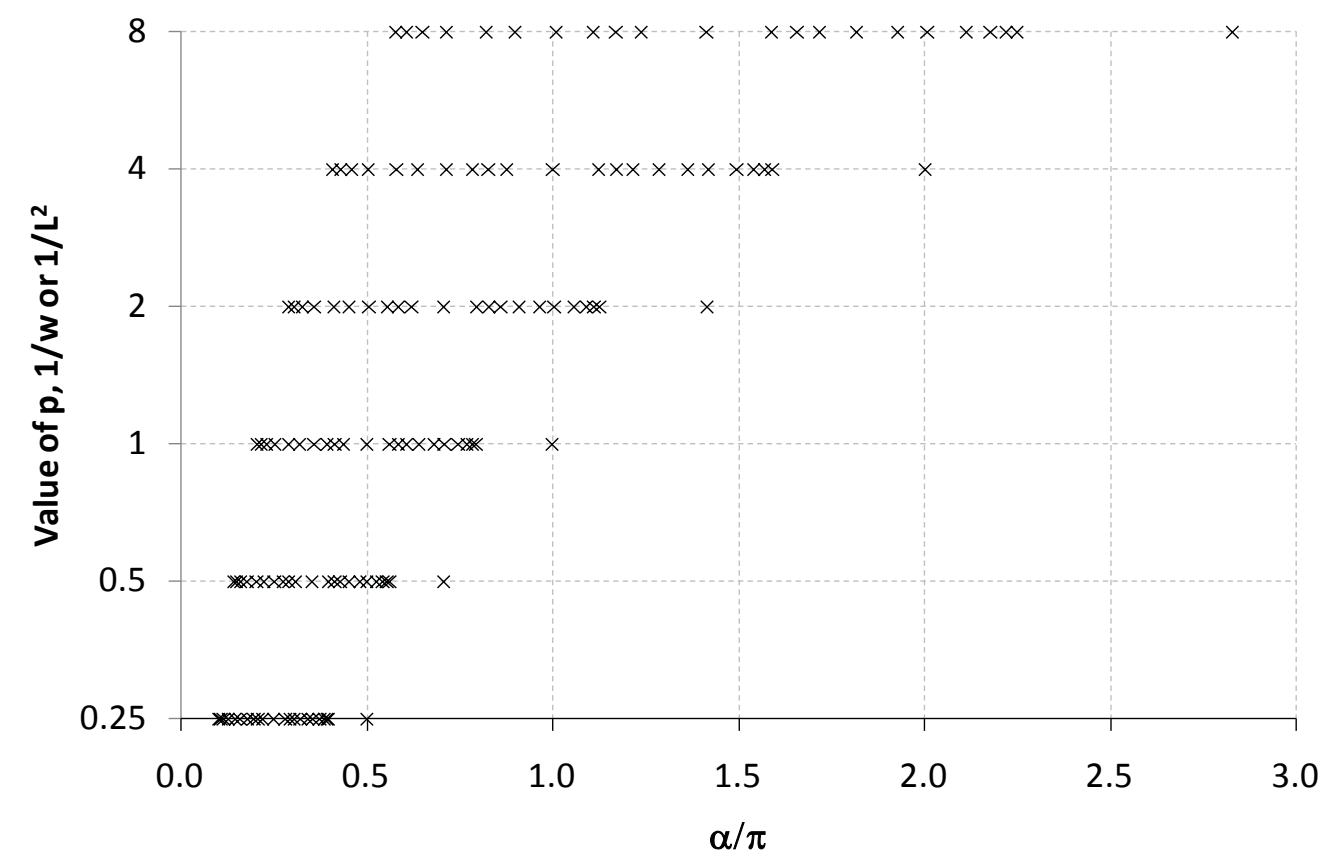

Figure 7: Effect of $L, p$ and $w$ on the eigenvalues of a tree with $b=3$ and $q=0$ 


\subsection{Alternative boundary conditions}

The root of the tree and the tips of the top branches can have any combination of Dirichlet $(y=0)$ or Neumann $(d y / d x=0)$ boundary conditions imposed upon them. The results for the Dirichlet / Dirichlet tree have been shown above. The same tree is now investigated for the remaining boundary conditions, subject to: $L=p=w=1 ; q=0 ; b=$ 3 and $n=8$. Table 2 shows the eigenvalues for all possible boundary conditions. It is partitioned into two sections to facilitate comparison of like results.

On the left hand side of the table, the Dirichlet / Dirichlet results (presented earlier) are compared with the equivalent Neumann / Dirichlet results. It can be seen that the spectrum of eigenvalues for these two trees are very similar, the only notable difference being that the trees do not share those eigenvalues with a multiplicity of 1 . It is interesting to note that the eigenvalues of both trees fall within the Sobolev and Solomyak bounds of 0.166666 and 0.833333 or at $\alpha=\pi$ and that the total number in each case is 3280. i.e. $1093+2187$ for the Dirichlet / Dirichlet tree and $1094+2186$ for the Neumann / Dirichlet tree. Such results suggest that when these trees are of infinite length they will share the same spectrum.

The Dirichlet / Neumann and the Neumann / Neumann trees that are compared on the right hand side of the table also have similar eigenvalues and retain the characteristic that the eigenvalues of multiplicity 1 are not shared. In addition, they clearly have eigenvalues that fall outside the Sobolev and Solomyak bounds, including a zero eigenvalue for the Neumann / Neumann tree, which corresponds to a rigid body mode in the structural mechanics analogy. Furthermore, it can be seen from the table that the Sobolev and Solomyak bounds occur in rows 8 and 34 and correspond to high 
multiplicity eigenvalues. The complete set of eigenvalues therefore fall either within the same band as the trees on the left hand side of the table or in the gaps between such bands. A band - gap spectra can therefore be postulated in which the eigenvalues that lie outside the band remain discrete and never form a continuous spectrum, while the remainder increasingly populate the band and form a continuous spectrum as the tree becomes infinitely long. Once more the total number of eigenvalues in the range $(0, \pi]$ is 3280 for both trees. This is exactly the same as the total number of eigenvalues calculated for the trees on the left hand side of the table. 
Table 2: Eigenvalues for the tree with $L=p=w=1 ; q=0 ; b=3$ and $n=8$

\begin{tabular}{|c|c|c|c|c|c|c|c|}
\hline \multirow[t]{2}{*}{$\begin{array}{l}\text { Eig. } \\
\text { No. }\end{array}$} & \multirow[t]{2}{*}{$\alpha / \pi$} & \multicolumn{2}{|c|}{$\begin{array}{l}\text { Boundary conditions } \\
\text { Tips = Dirichlet (D) }\end{array}$} & \multirow[t]{2}{*}{$\begin{array}{l}\text { Eig. } \\
\text { No. }\end{array}$} & \multirow[t]{2}{*}{$\alpha / \pi$} & \multicolumn{2}{|c|}{$\begin{array}{l}\text { Boundary conditions } \\
\text { Tips = Neumann }(\mathrm{N})\end{array}$} \\
\hline & & Root $=\mathrm{D}$ & Root $=N$ & & & Root $=\mathrm{D}$ & Root $=N$ \\
\hline 1 & 0.192764 & - & 1 & 1 & 0.000000 & - & 1 \\
\hline 2 & 0.204778 & 1 & - & 2 & 0.004544 & 1 & - \\
\hline 3 & 0.215086 & 2 & 2 & 3 & 0.007888 & 2 & 2 \\
\hline 4 & 0.230053 & 6 & 6 & 4 & 0.013739 & 6 & 6 \\
\hline 5 & 0.252903 & 18 & 18 & 5 & 0.024117 & 18 & 18 \\
\hline 6 & 0.260294 & - & 1 & 6 & 0.043118 & 54 & 54 \\
\hline 7 & 0.290215 & 55 & 54 & 7 & 0.080431 & 162 & 162 \\
\hline 8 & 0.318440 & 2 & 2 & 8 & 0.166667 & 486 & 486 \\
\hline 9 & 0.350019 & - & 1 & 9 & 0.204778 & - & 1 \\
\hline 10 & 0.357451 & 168 & 168 & 10 & 0.224337 & 1 & - \\
\hline 11 & 0.392474 & 1 & - & 11 & 0.242137 & 2 & 2 \\
\hline 12 & 0.413764 & 18 & 18 & 12 & 0.268468 & 6 & 6 \\
\hline 13 & 0.438273 & 2 & 2 & 13 & 0.290215 & - & 1 \\
\hline 14 & 0.449156 & - & 1 & 14 & 0.309216 & 18 & 18 \\
\hline 15 & 0.500000 & 547 & 546 & 15 & 0.328789 & 1 & - \\
\hline 16 & 0.550844 & - & 1 & 16 & 0.367531 & 2 & 2 \\
\hline 17 & 0.561727 & 2 & 2 & 17 & 0.376451 & 54 & 54 \\
\hline 18 & 0.586236 & 18 & 18 & 18 & 0.392474 & - & 1 \\
\hline 19 & 0.607526 & 1 & - & 19 & 0.421396 & 6 & 6 \\
\hline 20 & 0.642549 & 168 & 168 & 20 & 0.442329 & 1 & - \\
\hline 21 & 0.649981 & - & 1 & 21 & 0.500000 & 1640 & 1641 \\
\hline 22 & 0.681560 & 2 & 2 & 22 & 0.557671 & 1 & - \\
\hline 23 & 0.709785 & 55 & 54 & 23 & 0.578604 & 6 & 6 \\
\hline 24 & 0.739706 & - & 1 & 24 & 0.607526 & - & 1 \\
\hline 25 & 0.747097 & 18 & 18 & 25 & 0.623549 & 54 & 54 \\
\hline 26 & 0.769947 & 6 & 6 & 26 & 0.632469 & 2 & 2 \\
\hline 27 & 0.784914 & 2 & 2 & 27 & 0.671211 & 1 & - \\
\hline 28 & 0.795222 & 1 & - & 28 & 0.690784 & 18 & 18 \\
\hline 29 & 0.807236 & - & 1 & 29 & 0.709785 & - & 1 \\
\hline \multirow[t]{12}{*}{30} & 1.000000 & 2187 & 2186 & 30 & 0.731532 & 6 & 6 \\
\hline & & & & 31 & 0.757863 & 2 & 2 \\
\hline & & & & 32 & 0.775663 & 1 & - \\
\hline & & & & 33 & 0.795222 & - & 1 \\
\hline & & & & 34 & 0.833333 & 486 & 486 \\
\hline & & & & 35 & 0.919569 & 162 & 162 \\
\hline & & & & 36 & 0.956882 & 54 & 54 \\
\hline & & & & 37 & 0.975883 & 18 & 18 \\
\hline & & & & 38 & 0.986262 & 6 & 6 \\
\hline & & & & 39 & 0.992112 & 2 & 2 \\
\hline & & & & 40 & 0.995456 & 1 & - \\
\hline & & & & 41 & 1.000000 & - & 1 \\
\hline
\end{tabular}




\section{Conclusions}

The paper then shows how a structural mechanics approach can be used to solve a quantum graph problem. Theory has been presented that yields exact solutions to the Sturm-Liouville problem on homogeneous trees, together with an extremely efficient and compact computer program to implement it. The program is fully annotated and its use as a 'black box' is fully described. The program has been used to perform a parametric study to confirm previous results available in the literature and also to investigate parameter variations and the effect of boundary conditions. The former showed that the parameters associated with the Sturm-Liouville equation have considerable influence on the shape of the band, although the fundamental band-gap structure was unaffected for the boundary conditions considered. On the other hand, the investigation into the effect of the boundary conditions revealed a more fundamental issue. Initially it is shown that a tree with Dirichlet conditions at the tips of the branches had essentially the same band-gap spectrum regardless of the boundary condition at the root of the tree. However, when the Neumann condition was imposed at the tips, the band gap structure of the spectrum was retained, but discrete eigenvalues were introduced into the gaps. In the limit, as the tree becomes infinitely long, the band becomes fully populated and that there are no gaps in the spectrum. In addition it was shown that negative eigenvalues result from a sufficiently large value of the parameter q. The corollary of this is that for any problem with negative eigenvalues the negative eigenvalues can all be computed by shifting the eigenvalues so that they are all positive and computing these values. The value of the parameter $q$ can then be subtracted to reveal the true value of the eigenvalue. 
The code presented shows that a graph with a high degree of symmetry can be coded very easily and efficiently if the analysis methods presented are utilized. Other quantum graphs with high degrees of symmetry include lattice structures. Hence the study of photonic crystals could therefore be a useful area for future research by the authors or other researchers. Finally the dynamic stiffness method is a very useful tool for solving quantum graph problems 


\section{Appendix A}

\section{FORTRAN 77 computer program}

This program should always be run using double precision arithmetic or better.

1

2. $\operatorname{READ}\left(5,{ }^{*}\right) \mathrm{N}, \mathrm{NB}, \mathrm{IBC} 1, \mathrm{IBC} 2, \mathrm{JL}, \mathrm{JU}, \mathrm{CF} \quad$ ! Data input. See Table 1

3. $\quad$ WRITE(6,1000)N,NB,IBC1,IBC2,JL,JU,CF ! Echo print of data

4. $\mathrm{JR}=\mathrm{JL}-1$

! Initiate eigenvalue required

5. $10 \mathrm{JR}=\mathrm{JR}+1$

! Increment eigenvalue required

6. $\quad \mathrm{AU}=1 . \mathrm{E} 6$

7. $\mathrm{AL}=0.0$

! Set upperbound $\alpha$

8. $\quad \mathrm{AC}=1.0$

! Set lowerbound $\alpha$

9. $20 \mathrm{~J}=0$

! Set current $\alpha$

10. $\mathrm{AS}=0.0$

! Set eigenvalue counter

11. DO $40 \mathrm{~L}=1, \mathrm{~N}$

! Set subsystem parameter

12. $\mathrm{NE}=\mathrm{NB} * *(\mathrm{~N}-\mathrm{L})$

! Loop over vertices $\mathrm{I}=\mathrm{N}-\mathrm{L}$

13. $\mathrm{A} 1=\mathrm{COS}(\mathrm{AC}) / \mathrm{SIN}(\mathrm{AC})$

! No. of edges at current level

14. $\mathrm{A} 2=-1.0 / \mathrm{SIN}(\mathrm{AC})$

! Components of edge matrix

15. $\mathrm{A} 1 \mathrm{~S}=\mathrm{A} 1+\mathrm{AS}$

! See Eq.(11)

16. $\mathrm{J}=\mathrm{J}+\mathrm{NE} * \mathrm{INT}(\mathrm{AC} / \mathrm{PI})$

! Augment subsystem parameter

17. IF(L.EQ.1.AND.IBC2.EQ.0)GOTO 30

! $J_{e i}$ See Eq. (15)

18. IF(A1S.LT.0.0)J $=\mathrm{J}+\mathrm{NE}$

! Branch on boundary condition

19. $\mathrm{AS}=\mathrm{NB} *(\mathrm{~A} 1 * \mathrm{~A} 1 \mathrm{~S}-\mathrm{A} 2 * \mathrm{~A} 2) / \mathrm{A} 1 \mathrm{~S}$

! s $\left\{\mathbf{A}_{s}\right\}$ See Eqs.(12) and (17)

20. GOTO 40

! See Eqs.(17) - (20)

21. $30 \mathrm{AS}=\mathrm{NB} * \mathrm{~A} 1$

! See Eq.(16)

22. 40 CONTINUE

!

23. IF(IBC1.EQ.0)GOTO 50

! Branch on boundary condition

24. IF(AS.LT.0.0) J=J+1

! s\{A $\}$ See Eqs.(12) and (21)

25. $50 \mathrm{IF}\left(\mathrm{CF}^{*}(\mathrm{AC}-\mathrm{AL})\right.$.LE.AC)GOTO 100

! Branch if converged

26. IF(J.LT.JR) GOTO 60

! Set bounds on $\alpha$

27. $\quad \mathrm{AU}=\mathrm{AC}$

! Upper bound 
28. GOTO 70

29. $60 \mathrm{AL}=\mathrm{AC}$

30. IF(AU.LT.1.E5)GOTO 70

31. $\mathrm{AC}=2.0 * \mathrm{AC}$

32. GOTO 80

33. $70 \mathrm{AC}=0.5 *(\mathrm{AL}+\mathrm{AU})$

34. 80 IF(AC.LT.1.E-3)GOTO 90

35. GOTO 20

36. $90 \mathrm{AC}=0.0$

37. 100 WRITE(6,1010)JR,AC,AC/PI

38. IF(JR.LT.JU)GOTO 10

39. 9999 STOP

40. 1000 FORMAT(1X,6I4,1PE12.4/)

41. 1010 FORMAT(1X,I8,1P2E16.6)

42.

END
!

! Lower bound

! Set new trial $\alpha$

! Double if not bounded

!

! Interpolate if bounded

! Catch a zero eigenvalue

!

! Set output parameter

! Print solution

! Continue if in range 


\section{Appendix B}

\section{Data preparation and interpretation of results}

The data input for the program is very straightforward and is presented in Table 3. The output from the program comprises an echo print of the input data followed by a single line of results for each required eigenvalue, as described in Table 4.

Table 3: Data input scheme

\begin{tabular}{ccl}
\hline Line & Variable & Comment \\
\hline 1 & $\mathrm{~N}$ & Highest vertex level, $n$ \\
\hline \multirow{3}{*}{ NB } & Branching number, $b$ \\
\hline IBC1 & $\begin{array}{l}\text { Boundary conditions at the root of the tree. } \\
0=\text { Dirichlet (clamped), 1 = Neumann (free) }\end{array}$ \\
\hline IBC2 & $\begin{array}{c}\text { Boundary conditions at the top of the tree. } \\
\end{array}$ & $0=$ Dirichlet (clamped), 1 = Neumann (free) \\
\hline JL & Lowest value of $\alpha$ required \\
\hline JU & Highest value of $\alpha$ required \\
\hline CF & Convergence factor. $\alpha$ is found to 1 part in CF \\
\hline
\end{tabular}

Table 4: Output results

\begin{tabular}{cll}
\hline Line(s) & Variable & Comment \\
\hline 1 & & Echo print of the input data \\
\hline Subsequent & JR & Eigenparameter number \\
\hline & AC & $\alpha$ \\
\hline & AC/PI & $\alpha / \pi$
\end{tabular}


In order to consolidate the input/output scheme, a data file that will determine the first ten values of $\alpha$ for the three level, binary tree of Figure 3 is given in Table 5. The corresponding output file is given in Table 6.

Table 5: Data file for the three level, binary tree of Figure 3 $321101101 . e 7$

Table 6: Results file for the three level, binary tree of Figure 3

\begin{tabular}{|c|c|c|c|}
\hline 3 & 2 & $\begin{array}{lll}0 & 1 & 10\end{array}$ & $1.0000 \mathrm{E}+\odot 7$ \\
\hline & 1 & 7. 297277E- $\odot 1$ & $2.322795 E-01$ \\
\hline & 2 & $1.570796 \mathrm{E}+\odot \odot$ & $5.000 \odot \odot \odot E-\odot 1$ \\
\hline & 3 & $1.570796 \mathrm{E}+\odot \odot$ & $5.000 \odot \odot \odot E-\odot 1$ \\
\hline & 4 & $2.411865 \mathrm{E}+\odot \odot$ & $7.677205 E-01$ \\
\hline & 5 & $3.141593 \mathrm{E}+\odot \odot$ & 1. $.00 \odot \odot \odot E+\odot \odot$ \\
\hline & 6 & $3.141593 \mathrm{E}+\odot \odot$ & 1. $.00 \odot \odot \odot E+\odot \odot$ \\
\hline & 7 & $3.141593 \mathrm{E}+\odot \odot$ & 1. $.000000 \mathrm{E}+\odot \odot$ \\
\hline & 8 & $3.871320 \mathrm{E}+\odot \odot$ & $1.232280 E+\odot \odot$ \\
\hline & 9 & $4.712389 \mathrm{E}+\odot \odot$ & $1.500000 \mathrm{E}+\odot \odot$ \\
\hline & 10 & $4.712389 \mathrm{E}+\odot \odot$ & $1.500000 \mathrm{E}+\odot \odot$ \\
\hline
\end{tabular}




\section{References}

[1] Peter Kuchment, “Quantum graphs: an introduction and a brief survey”, Math Sci Net, arXiv:0802.3442v1 [math-ph] 23 Feb 2008

[2] W.D. Evans, D.J. Harris, “Fractals, trees and the Neumann Laplacian”, Math. Annalen, 296(3), 493-527, 1993.

[3] W.D. Evans, D.J. Harris, J. Lang, “The approximation numbers of Hardy-type operators on trees”, Proc. London Math. Soc (3), 83(2), 390-418, 2001.

[4] A.V. Sobolev, M. Solomyak, "Schrödinger operators on homogeneous metric trees spectrum in gaps”, Reviews in Mathematical Physics 14(5), 421-467, 2002.

[5] E.A. Coddington, N.Levinson, “Theory of ordinary differential equations”. New York; McGraw-Hill, 1955.

[6] M. Marletta, J.D. Pryce, “A new multipurpose software package for Schrodinger and Sturm- Liouville Computations”, Comp. Phys. Comms., 62(1), 42-52, 1991.

[7] M. Marletta, “Numerical Solution of Eigenvalue Problems for Hamiltonian Systems”, Adv. Comp. Maths., 2(2), 155-184, 1994.

[8] S. Pruess, C.T. Fulton, Y. Xie, “An asymptotic numerical method for a class of singular Sturm Liouville problems”, SIAM J Num. Anal., 32(5), 1658-1676, 1995. [9] P.B. Bailey, W.N. Everitt, A. Zettl, “The SLEIGN2 Sturm-Liouville Code”, ACM Transactions on Mathematical Software, 27, 143-192, 2001.

[10] B.M. Brown, D.K.R. McCormack, M. Marletta, “Guaranteed error bounds for eigenvalues of singular Sturm-Liouville problems”, Math. Nachrichten, 213, 17-33, 2000. 
[11] R. Carlson, “Hill’s equation for a homogeneous tree”, Electron. Journal of differential Equations, 23, 1-30, 1997.

[12] F.W. Williams, W.P. Howson, A. Watson, “Application of the Wittrick-Williams algorithm to the Sturm-Liouville problem on homogeneous trees: A structural mechanics analogy”, Proceedings of the Royal Society series A: Mathematical, Physical and Engineering Sciences, 460(2045), 1243-1268, 2004.

[13] F.W. Williams, A. Watson, W.P. Howson, A.J. Jones. “Exact solutions for SturmLiouville problems on trees via novel substitute systems and the Wittrick-Williams algorithm”, Proceedings of the Royal Society series A: Mathematical, Physical and Engineering Sciences, 463(2088), 3195-3224 2007.

[14] V. Kostrykin, R. Schrader, "Laplacians on Metric Graphs: Eigenvalues, Resolvents and Semigroups”, Contemporary Mathematics, 201-225, 415, 2006 [15] J. Behrndt, A. Luger, “On the number of negative eigenvalues of the Laplacian on a metric graph”, J. Phys. A: Math. Theor. 43, 1-11, 2010.

[16] G. Berkolaiko, P. Kuchment, "Dependence of the Spectrum of a Quantum Graph on Vertex Conditions and Edge Lengths”, arXiv:1008.0369v2 [math-ph] 10 ${ }^{\text {th }}$ Feb 2011 Proceedings of the

[17] A. Watson, W.P. Howson, “Computation of Lower Bound Eigenvalues using the Wittrick-Williams Algorithm”, 8th International Symposium on Vibrations of Continuous Systems, Whistler, Canada, July 18-22, 2011 


\section{Figure Captions}

Figure 1: Graph (a) Non-cyclic graph (tree); (b) Cyclic graph.

Figure 2: The topology of typical trees showing edge and vertex levels together with their branching number

Figure 3: A three level, binary tree in which one of the two most deeply nested subtrees at edge level $i=2$ is annotated and represents the start point for analysis.

Figure 4: The first five repeating portions of the infinite band-gap spectra for the family of trees shown in Figure 2 when all edges are identical. The Sobolev and Solomyak bounds are shown dashed [3].

Figure 5: Discrete eigenvalues for a tree with $b=3 ; L=p=w=1 ; q=0$ and Dirichlet $(y=0)$ boundary conditions at each end of the tree. The two solid lines SSL and SSU are theoretical lower/upper bounds, respectively, obtained by Sobolev and Solomyak [4]

Figure 6: Effect of varying $q$ on the eigenvalues of a tree with $b=3$ with $L=p=w=1$ and Dirichlet / Dirichlet boundary conditions [17]. The abscissa values to the left of the origin are imaginary numbers and hence need to be multiplied by $-\sqrt{ }(-1)$.

Figure 7: Effect of $L, p$ and $w$ on the eigenvalues of a tree with $b=3$ and $q=0$

\section{Table Captions}

Table 1: Numerical values of the normalised eigenvalues described in Figure 5, together with their multiplicities

Table 2: Eigenvalues for the tree with $L=p=w=1 ; q=0 ; b=3$ and $n=8$

Table 3: Data input scheme

Table 4: Output results

Table 5: Data file for the three level, binary tree of Figure 3

Table 6: Results file for the three level, binary tree of Figure 3 